\title{
LES USAGERS FACE AUX MÉDIAS : DE LA RÉTROACTION AU PACTE
}

\author{
Frédéric Antoine ${ }^{1}$
}

\section{L'usager dans les sciences de la communication}

Il y a une quinzaine d'années déjà, dans leur ouvrage Penser les médias, Armand et Michèle Mattelart constataient qu'on assistait alors, au sein des sciences de la communication, à une véritable "réhabilitation du sujet". Ils écrivaient à ce propos : "Les périodes de croissance et les idéologies qui les accompagnent favorisent-elles l'oubli du récepteur, du consommateur et du citoyen ? Contribuentelles à entretenir l'illusion que l'on peut se passer d'eux et planifier leurs demandes au gré des bienfaits de la redistribution? À la lumière de ce qui se passe aujourd'hui, on est tenté de le croire. Il a fallu attendre l'entrée dans la crise pour voir légitimée enfin l'idée pourtant élémentaire que le processus de communication se construit grâce à l'intervention active d'acteurs sociaux très divers"'.

À cette époque en effet, la recherche commençait enfin à se préoccuper de l'usager des médias, dont les Mattelart annonçaient ainsi "le retour".

1 Professeur au Département de communication de l'Université catholique de Louvain.

2 A. et M. Mattelart, Penser les médias, Paris, La Découverte, 1986, p. 97.

Recherches en communication, $\mathrm{n}^{\circ} 21$ (2004). 
Mais s'agissait-il vraiment d'un véritable "retour" ? Ou, depuis la naissance des premiers modèles communicationnels, ne s'était-on jamais totalement préoccupé de l'usager des médias, sinon en tant qu'ultime maillon faible d'un processus auquel échappait toute maîtrise?

Faut-il rappeler ici ${ }^{1}$ la place accordée (on aurait tendance à écrire "concédée") au récepteur dans les premières modélisations du processus de communication, telle la théorie mathématique de l'information élaborée par Shannon et Weaver en 1949 ? Se contentant de le représenter de manière linéaire et unidirectionnelle, ils valorisèrent un dispositif dans lequel le rôle essentiel était attribué au pôle "émission" tandis que la fonction la plus obscure et la plus passive revenait au pôle "réception". En effet, "s'il éclaire bien la stratégie de l'émetteur ou le codage du message, [ce modèle théorique] a son point aveugle du côté du récepteur. [Car] le modèle du transfert bloque les publics et les auditoires des médias -d'ailleurs désignés du mot redoutable de "récepteurs" qu'ils partagent avec les combinés téléphoniques et les postes de télévision- dans une position fondamentale de passivité. Seuls les émetteurs sont des acteurs sociaux. Les récepteurs sont a priori privés d'initiatives et de stratégies, incapables de créer du sens (se bornant à enregistrer, à déformer ou à rejeter, celui qu'a fabriqué l'émetteur). Ils ne sont saisis que comme la cible visée"2.

Il faudra qu'on rompe "avec le schéma stimulus-réponse" 3 et qu'émerge dans le champ des théories communicationnelles la notion de rétroaction, inspirée par la cybernétique, pour que l'on se préoccupe aussi, dans le secteur de la communication, de ce "concept clé"4 qu'est le feedback, et donc du pôle "réception". Dans la foulée de Norbert Wiener, on se mettre alors à envisager la communication comme un processus unique, circulaire, où des informations sur l'action en cours nourrissent en retour le système et lui permettent d'atteindre son but. À ce stade, la rétroaction sera dès lors définie comme une boucle récursive d'information, la circulation des

\footnotetext{
1 Précisons pour le lecteur spécialiste que l'intention de ce texte n'étant pas de traiter en profondeur des théories de la communication, nous nous contenterons ici de brefs rappels généraux, qui paraîtront peut-être sommaires aux théoriciens de la science communicationnelle, mais qui n'ont comme intention que de contextualiser le propos que nous développerons ensuite.

2 J. BianchI, H. Bourgeors., Les médias côté public, Paris, Centurion, 1992, pp. 34 35.

3 D. Bougnoux, La communication par la bande, Paris, La Découverte, 1992, p. 11.

4 Ibid., p. 192.
} 
informations nécessaires devant "former une boucle fermée dans laquelle la machine évalue les effets de ses actions et corrige son comportement futur en utilisant les performances passées"'.

La cybernétique soulignera aussi l'importance de la rétro-information, de la nécessaire circularité des processus dynamiques dans l'analyse du fonctionnement de tout système,

Un pas supplémentaire sera franchi grâce à Bateson, qui dégagea les notions de feedback positif et négatif. Selon cet anthropologue, la rétroaction positive conduit les éléments d'un système à accentuer un phénomène ou un changement. Poussée à son paroxysme, elle peut renforcer la société dans son escalade vers la destruction totale. Le feedback négatif désigne par contre les autorégulations successives par lesquelles le système est capable de retourner à l'état de stabilité (homéostasie). Au contraire du feedback positif, la rétroaction négative tend donc à amortir tout phénomène de changement, cette forme de rétroaction constituant un mécanisme de régulation tendant à conserver le système dans un état donné. Dans ce cadre, la stabilité d'un système reposera dès lors sur un couplage des deux types de feedback : le feedback négatif, qui désigne l'information qui permet au système de maintenir entre ses éléments des relations stables, et le feedback positif, qui conduit au changement, à la perte de stabilité ou d'équilibre.

À la suite de Bateson, les notions de rétroaction positive et négative sont devenues centrales pour comprendre comment tout système (individuel, social, économique...) noue avec son environnement des interactions dont dépendent sa survie ou sa mort, son éclatement ou son expansion.

Appliquées au modèle communicationnel, ces notions et leur inscription dans un cadre d'analyse systémique permettaient enfin de prendre en considération le pôle "destinataire" d'un autre point de vue que de celui de simple agent ultime d'un processus linéaire.

L'apport des théories systémiques, considérant que "toute communication présente deux aspects : le contenu et la relation" (Watzlawick) a aussi révolutionné les visions classiques du processus de la réception, dont le seul angle d'approche du problème consista longtemps à "la regarder depuis l'émetteur à travers la grille behavioriste et fonctionnaliste des "effets" produits"2. Désormais, on

I M. Edmont, D. Picard, L'école de Palo Alto, Paris, Retz, 1984, p. 20.

2 J. BIANCHI, H. BOURGEOIS, op. cit., p. 35. 
considérera que "médias et récepteurs sont en situation de causalité circulaire. Leurs rapports ne peuvent plus être ramenés à l'action d'une instance sur l'autre, mais doivent être compris comme leur ajustement, permanent et réciproque, dans un contexte précis. La réception rétroagit sur l'émission, des règles gouvernent leurs échanges" 1 .

Les récentes approches de la réception centrées sur l'étude des usages n'ont fait que confirmer cette nouvelle reconnaissance du statut d' "usager" aux utilisateurs des supports de communication médiatique. D’objet, celui-ci est devenu sujet.

Car il est désormais clair que "le récepteur génère du sens" et "ne se borne pas à recueillir le sens codé dans le média"2. "Chaque fois qu'il y a reprise en charge du texte par le lecteur, il y a chance qu'émerge le rôle du producteur de sens"'s.

L'approche de l'analyse du récit médiatique cultivée au sein de l'équipe de l'Observatoire du Récit médiatique (ORM) du département de communication de l'Université catholique de Louvain ne dit pas autre chose lorsqu'elle s'inspire, pour appréhender son objet d'étude, de la boucle mimétique théorisée par Paul Ricœur. À la suite de l'auteur dans les trois tomes de Temps et récit, la lecture du phénomène médiatique en une triple mimésis (figuration, configuration, refiguration) n'impose pas seulement de prendre en considération le moment où le récit est refiguré dans le chef de celui qui le lit, le voit ou l'entend. Plus fondamentalement, cette approche trouve en fait sa seule raison d'être dans l'existence de ce troisième moment de la boucle. Car "le travail de la pensée à l'œuvre en toute configuration narrative s'achev[ant] dans une refiguration de l'expérience temporelle"4, sans refiguration, le récit (et, partant, le mécanisme communicationnel dans lequel il s'inscrit) n'aurait ni de raison ni de possibilité d'être. La refiguration peut ainsi être identifiée au moment "de la référence croisée entre histoire et fiction", le temps humain procédant de cet entrecroisement "dans le milieu de l'agir et du souffrir"s.

Loin d'une simple composante d'un mécanisme de feedback, le "refigurateur" humain du récit en vient à occuper une place centrale

1 Ibid., p. 38.

2 Ibid., p. 39.

3 L. BAZINET Le lecteur comme écrivain, Montréal, Novotexto-Aupelf, 1990, p. 57.

4 P. Ricceur, Le temps raconté, Paris, Éd. du Seuil, 1985, p. 9.

5 Ibid., p. 147 (Ricour y fait référence au Tome 1 de Temps et récit, p. 109 sq.) 
dans le processus communicationnel. C'est, en définitive, lui qui, donnant sens au texte, procure également sens à l'ensemble du processus.

Plus que jamais, il convient donc de se mettre à l'écoute de l'usager, lui que Le Robert désigne comme étant celui "qui a un droit réel d'usage", c'est-à-dire "le fait de faire agir (un objet) pour obtenir un effet qui satisfasse un besoin".

\section{L'usager et les médias}

Cela étant posé, il nous semble qu'une réelle reconnaissance du rôle de l'usager dans le processus communicationnel ne peut se satisfaire du constat selon lequel ce dernier serait aujourd'hui revenu, sinon au centre, du moins parmi les objets d'étude de prédilection des chercheurs en communication. Dans une société en crise, pour reprendre l'intuition de Mattelart évoquée au début de ce texte, et où les références à la citoyenneté se font plus pressantes depuis quelques années, il paraît opportun de s'interroger sur la nature du statut que les entreprises de communication et d'information, lieux de matérialisation du processus médiatique, confèrent aujourd'hui à leurs "usagers"'.

Car, en tant que systèmes finalisés, les médias ne peuvent échapper aux phénomènes de rétroaction. Comme le rappelle Michel Mathien, au même titre que tout système soumis à rétroaction, les systèmes médiatiques sont influencés par leur comportement passé, leur histoire, les buts qu'ils cherchent à atteindre et leurs relations à l'environnement. L'entreprise médiatique est prise dans un cycle d'échange avec son environnement. En fonction de ces éléments, les processus de rétroaction ne peuvent qu'y jouer un rôle prépondérant ${ }^{2}$.

Dans ce cadre, les "usagers" des organes d'information sont-ils reconnus comme tels, les médias s'inscrivant à ce propos dans ce

1 Une étude en ce sens, reposant sur une interrogation concernant l'émergence d'une "nouvelle citoyenneté" au travers des modes d'interactions entre médias et usagers survenus notamment lors des "affaires" de mort d'enfants qui ont touché la société belge au milieu des années '90 a été ménée par l'ORM grâce à l'appui du Fonds national de la Recherche scientifique (FNRS). Certains éléments issus de ce travail seront présentés dans ce dossier sous la plume des trois chercheurs qui prirent en charge ce travail à l'ORM sous la direction de Frédéric Antoine.

2 M. MATHIEn,Le système médiatique, le journal dans son environnement, Paris, Hachette, 1989, p. 22. 
courant de "retour de l'usager" que nous pressentons à l'œuvre dans les sciences de la communication ? Ou, les considérant davantage comme des clients "consommateurs" que comme des utilisateurs, se contentent-ils de les maintenir à distance, reproduisant en quelque sorte à leur égard le schéma primaire de la communication décrit précédemment et s'inscrivant, en fin de compte, dans la simple continuité d'une logique historique implacable?

On connaît aujourd'hui les théories sur le principe de publicité (Öffentlichkeit) et l'émergence de l'espace public dans les sociétés bourgeoises, dont Jürgen Habermas fut le premier concepteur. On sait aussi le rôle que ce dernier conféra à ce propos aux médias du XVIII siècle, c'est-à-dire à la presse, considérée comme le lieu du débat, la zone neutre d'échange pour le bien commun, mais aussi l'espace de médiation alors indispensable au cœur de la société1. Comme "la presse quotidienne produi[sait alors] l'opinion en la rapportant", et comme "elle la rév[élait] en la faisant" 2 on aurait pu croire que, dès cette période, médias et autorités publiques auraient naturellement porté attention à ce "public", à la fois "usager" du produit médiatique mais aussi producteur des opinions qu'il véhiculait. On eût pu imaginer qu'organes de presse et composantes du pouvoir se soient préoccupés de ceux-là même que les États redoutaient de voir (ré)agir suite à la lecture des messages contenus dans les médias. En fin de compte, n'était-ce pas de ce "public" que les moyens de communication tiraient leur légitimité ? N'était-il pas le vrai détenteur du pouvoir de la presse?

Pourtant, dès cette période, on ne se préoccupa pas du public des usagers des médias. Comme le détermineront plus tard les modélisations du schéma de la communication, ni les médias ni les pouvoirs publics ne se soucièrent des droits des lecteurs, usagers ou "consommateurs" de médias. Seul préoccupa l'acte d'expression et le "contre-pouvoir" que pouvaient incarner les médias.

Longtemps, l'acte de "communiquer" ne fut ainsi assimilé qu'à un droit, lié à une capacité d'expression. C'est-à-dire à la liberté, accordée à une personne ou un groupe d'individus, de pouvoir manifester, de manière large, des opinions, des avis, en recourant dans ce but au support d'un moyen de communication. Les constitutions et déclarations sur lesquelles se fondent les sociétés démocratiques

I J. Habermas, L'espace public, Paris, Payot, 1978.

2 D. Bougnoux, op. cit., p. 161. 
modernes considèrent ainsi les médias comme un des lieux de l'exercice d'un des droits inaliénables des peuples. En Belgique, l'article 18 de la Constitution du 7 février 1831 affirme que "la presse est libre" et que "la censure ne pourra jamais être établie", après avoir proclamé dans des articles précédents la liberté des cultes et de l'enseignement, et avant de consacrer d'autres articles au droit de rassemblement, d'association et de pétition.

En France, l'article 11 de la Déclaration des droits de l'homme et du citoyen, adoptée le 24 août 1789, indiquait que "la libre communication des pensées et des opinions est un des biens les plus précieux de l'homme [et que] tout citoyen peut donc parler, écrire, imprimer librement, sauf répondre de l'abus de cette liberté dans les cas déterminés par la loi". Deux ans plus tard, le premier amendement de la Constitution américaine, si souvent évoqué depuis lors, affirmera que "le Congrès ne fera aucune loi (...) qui restreigne la liberté de parole ou de la presse".

Dans chacun de ces cas, "la presse", c'est-à-dire le moyen de diffusion à large échelle de l'époque, était considérée comme le lieurelais de l'exercice d'une liberté publique. Une liberté qui conférera par la suite aux médias ce "pouvoir" que l'historien Thomas Carlyle qualifia de "quatrième" en reprenant la formule de "quatrième État" utilisée au XVIII e siècle par l'orateur Edmund Burke lorsqu'il constata qu'aux côtés des trois États participant au Parlement anglais (les clercs, l'artistocratie et les "commons", ou tiers-État), il y siégeait un quatrième : celui qui demeurait dans la "Reporters's gallery" du parlement de Londres. Un État qui s'avérait même plus important que les trois autres réunis, car il possédait la possibilité d'écrire et d'imprimer. "Quiconque a la possibilité de s'adresser à la nation entière détient un immense pouvoir", avait remarqué Carlyle. Toutefois, la considération dont pouvait bénéficier cette "nation", ou les publics qui la composaient, resta (et reste encore) plus que restreinte.

Tout au long de leur histoire, les médias d'information n'ont cessé de reproduire un schéma communicationnel univoque et unidirectionnel dans lequel le pôle émetteur s'est rapidement arrogé le premier et quasiment seul rôle et s'est soucié de tout mettre en œuvre pour le conserver. Une lecture transhistorique des interactions entre 
médias et usagers est encore à construire'. Mais il est patent que, depuis qu'ils se sont institutionnalisés, les médias ont cultivé vis-à-vis de leurs usagers une prudente distance, qu'illustre à suffisance le confinement à un statut de "consommateurs" qui leur fut le plus souvent conféré. Reposant sur une logique de l'échange, qui se manifeste le plus souvent sous le mode d'une transaction financière (directe ou indirecte), ce statut évite de concéder à l'utilisateur du produit médiatique le moindre rôle rétroactif vis-à-vis du produit luimême.

Alors que, comme nous venons de l'évoquer, les médias tiennent leur légitimité sociale du nombre, du statut et de l'agir potentiel de leurs "utilisateurs", ils paraissent avoir toujours éprouvé une crainte certaine à voir leurs destinataires se préoccuper trop directement de leurs contenus et de leurs modes de fonctionnement et, a fortiori, interférer directement avec eux.

La culture journalistique, fondée sur la tradition de la toute puissance du pôle "production" du message, entretient chez les membres de cette profession la conviction que seul ce pilier du système communicationnel détient la connaissance et la vérité et que, étant à l'origine de la chaîne, il en est le seul maître et le seul détenteur de l'agenda.

\section{Les modes d'interactions médiatiques}

Néanmoins, comme il est désormais acquis que le média opère sous la configuration d'un système, il n'est pas étonnant qu'il se voit vu contraint de mettre en place des lieux ou interfaces de réception des feedback d'informations issues du corps de ses usagers.

Des inventaires de ces types de rétroactions ont d'ores et déjà été dressés et leurs modes de fonctionnement étudiés, nous aurons à y revenir.

Partant de l'énumération dressée à ce sujet par Claude-Jean Bertrand, et sur laquelle nous aurons également à nous prononcer dans la suite de ce texte, il nous a été donné de mener au cours de l'année 2000, dans une cadre d'une approche tendant à revaloriser le

\footnotetext{
1 Au travers de la recherche soutenue par le FNRS et évoquée précédemment, on a commencé à s'y employer au sein de l'Observatoire du récit médiatique de l'UCL. Le chantier est loin d'être terminé. À l'ORM, il est toutefois aujourd'hui en jachère, suite à l'arrêt du soutien d'organismes officiels.
} 
rôle de l'usager, une recherche appliquée sur les mécanismes de feedback à l'œuvre dans les médias belges ${ }^{1}$. Confrontation cette situation locale à quelques exemples étrangers particulièrement remarquables, cette étude nous a permis de dresser une liste concrète des modes d'interactions les plus significatifs.

Au terme de l'analyse, il nous a été permis de distinguer divers types de mécanismes de feedback (pour une énumération complète : voir tableau) et de les représenter en un schéma global (voir graphique). Les uns, "internes au média", découlent de l'univers de l'entreprise médiatique. Les autres, externes, relèvent de la société civile ou de l'initiative des usagers².

\section{- Univers interne au média}

- Initiatives émanant du média

- Études marketing : focus groups, enquêtes longitudinales...

- Création de "clubs de lecteurs"

- Initiatives émanant entre autres de la rédaction :

- Rubrique de courrier des lecteurs, séquence des auditeurstéléspectateurs

- Dialogue sur sites Internet

- Médiateur, ombusdman,

- Rencontres de lecteurs

- Invitation d'usagers à participer à l'élaboration d'une partie du produit médiatique

\section{- Univers des usagers directs}

- Initiatives émanant des usagers :

- Appels téléphoniques, courrier, faxs, emails aux médias

- Création d'associations citoyennes

- Création de médias "qui appartiennent à leurs lecteurs"

1 F. Antoine, M. HaRzimont, Le feedback des usagers dans les médias, Bruxelles, Fondation roi Baudouin, 2000. Certains éléments de cet article sont directement inspirés par les résultats de ce travail ainsi que par le questionnement qu'il a suggéré à ses auteurs.

2 Nous nous contenterons de présenter ici les grandes lignes des catégorisations provenant de ce travail de terrain. Pour davantage de précisions, le lecteur est invité à consulter le rapport publié à l'issue de cette étude, dont les références figurent cidessus. 


\section{- Univers de la sphère publique}

- Initiatives prises par les instances politiques

- Création d'instances de régulation et de contrôle

- Initiatives prises par les usagers et/ou instances politiques

- Création de "conseils d'usagers"

- Initiatives prises par les usagers et/ou instances politiques

- Conseils de presse

À l'intérieur du média, une série de systèmes rétroactifs constituent des initiatives propres au média comme entreprise : les études socio-économiques, les analyses d'audience et de lectorat y contribuent largement. De même que diverses actions commerciales tendant à conférer au "consommateur" du média un statut de "client privilégié" au sein de l'entreprise.

D'autres initiatives prises par certaines composantes de l'entreprise médiatique relèvent davantage d'une catégorisation du média comme lieu de communication et touchent au domaine des contenus. On retrouvera ici l'institutionnalisation des lieux d'échange internes entre "émetteur" et "récepteur" que constituent les rubriques de "courrier des lecteurs" et leurs diverses variantes spécifiques à chaque type de média (même si, dans ce cadre, l'initiative du contact revient à l'usager et non au média, cf. infra), ainsi que l'ensemble des interactions permises suite à l'émergence des nouveaux types de communications interactives qu'offre l'Internet. De manière plus globale, on notera aussi ici le rôle rempli par les organes de médiation internes aux médias, ainsi que les divers types de contacts directs mis en œuvre avec les usagers en lien avec les contenus que les médias proposent.

À côté de l'ensemble de ces modes de rétroaction suscités par les médias, il faut évidemment préciser que les usagers sont eux-mêmes à la source d'une série d'interactions directes avec les entreprises médiatiques qu'ils interpellent par courrier, télécopies, emails et appels téléphoniques. Avec la vulgarisation des moyens de communication électronique, et en particulier de l'Internet, ces types d'échanges ne cessent de se multiplier, au risque de les voir inonder les médias. 
Dans ce cadre, il vaut la peine d'accorder une attention particulière au courrier adressé aux médias par leurs lecteurs. L'existence du "courrier des lecteurs", bien antérieure à l'institutionnalisation de la sphère médiatique, fait sans doute de ce type de feedback le mode d'interaction le plus ancien entre les usagers et les médias. Il se présente aussi comme un mécanisme de rétroaction particulièrement intéressant à plusieurs titres. Initiative relevant des usagers euxmêmes, il revendique du média une reconnaissance spécifique du pôle des usagers, ainsi que l'établissement d'une plate-forme de dialogue entre acteurs du processus communicationnel. À certaines époques, il semble même avoir contribué à établir des périodes de coconstruction du message médiatique à la fois par les "professionnels" du secteur, dont relevait "normalement" l'émission de contenus médiatiques, et par une partie de la composante du pôle usagers. À ce titre, l'histoire du "courrier des lecteurs", à elle seule, mériterait une approche spécifique. Mais ce dernier ne constitue pas le seul mode de feedback dont l'initiative revient aux "utilisateurs" du média.

Certains usagers, plus actifs encore, n'hésitent pas à créer des associations destinées à interpeller ou à assurer leur prise en compte dans les médias.

D'autres ont parfois rêvé d'inverser totalement l'ordre communicationnel en concevant des médias qui appartiendraient à leurs lecteurs. Mais leurs efforts n'ont pas souvent été couronnés de succès. 


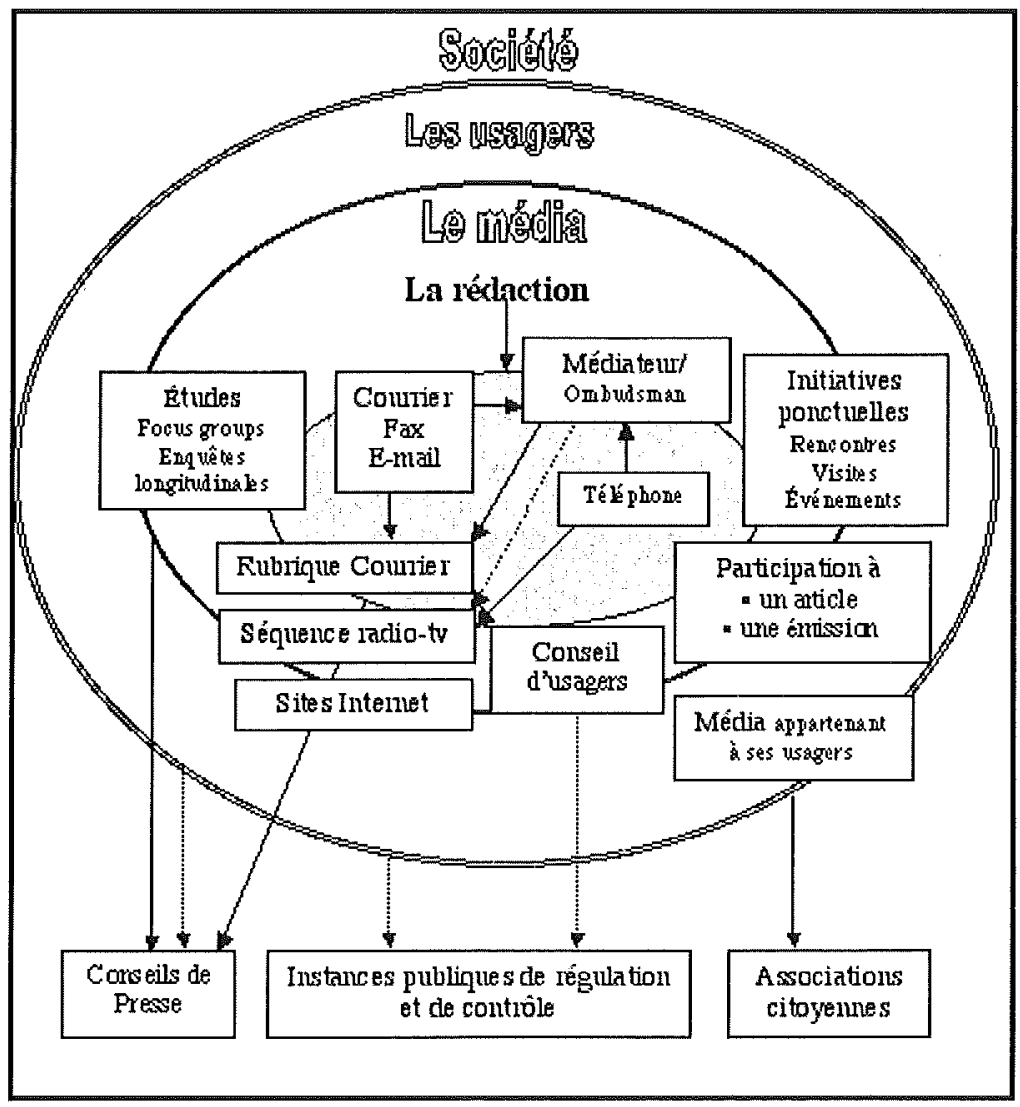

Schéma extrait de : F. ANTOINE, M. HARZIMONT, Le feedback des usager's dans les médias, Bruxelles, Fondation roi Baudouin, 2000, p. 137.

Enfin, la société elle-même estime parfois utile afin de faire entendre la voix des usagers vis-à-vis des médias en obligeant les uns et les autres à dialoguer au sein d'instances ad hoc. Ou en établissant, au nom des usagers, des modalités de contrôle de certains types de médias. 


\section{Rétroactions instrumentales et instrumentalisées}

Une constatation s'impose à l'issue de l'élaboration du tableau de modes de feedback des usagers dans les médias que nous venons de dresser.

Édifiés au sein de l'entreprise médiatique elle-même, bon nombre de ces mécanismes de rétroaction n'ont souvent été conçus que pour maintenir les systèmes-médias en état d'homéostasie ou pour tenter de rétablir un équilibre rompu suite aux évolutions des configurations du "marché" des "consommateurs" ou en réponse à des dysfonctionnements internes à l'entreprise.

Initiés par les médias afin d'améliorer leur adéquation à leur environnement, la plupart de ces mécanismes de feedback peuvent ainsi être considérés comme "instrumentaux", dans la mesure où leur unique mission revient à servir les intérêts de l'entreprise médiatique qui les a mis en œuvre.

Jusqu'à présent, leur observation par le chercheur en sciences de la communication s'est, le plus généralement, inscrite dans le cadre d'une une appróche spécifique du pôle "émetteur" et, plus particulièrement, dans le.contexte d'études concernant les pratiques journalistiques et des systèmes de régulation de cette profession ${ }^{1}$.

Dans le cadre d'interrogations globales sur la responsabilité sociale des médias et, en particulier, des journalistes, ces modes de rétroaction ont souvent été considérés comme des moyens permettant d'apprécier le fonctionnement de l'entreprise médiatique et d'évaluer ses capacités de pallier ses dérèglements (manières par lesquelles les médias entendent améliorer leur "produit", réagissent aux "plaintes" de leurs usagers ou établissent en veillaient en leur sein au respect de règles déontologiques et professionnelles). C'est dans cette perspective que s'inscrit la notion de $M^{*} A * R * S *$ (Moyens non-étatiques d'Assurer la Responsabilité Sociale des médias) imaginée par le chercheur français Claude-Jean Bertrand, qui en a établi un inventaire qu'il ne cesse de compléter d'année en année. L'auteur est clair à ce propos lorsqu'il écrit :

S'ils veulent accomplir leur mission, participer diversement au mieux-être de la société, les responsables de l'information publique doivent instaurer un contrôle de qualité dans leurs

1 Voir notamment : "L'autorégulation des journalistes ", sous la direction de Benoît GREVISSE, Recherche en communication, $n^{\circ}$ 9, Louvain-la-Neuve, 1998. 
journaux, magazines, radios, télévisions, sites Internet. Ils doivent faire en sorte que ces organes soient d'abord des services publics et, ensuite, des machines à faire des sous. En d'autres termes, ils doivent assumer leur "responsabilité sociale", au double sens que les médias doivent répondre aux besoins des usagers et qu'ils doivent être responsables envers eux, leur rendre des comptes ${ }^{1}$.

Bon nombre des modes de rétroaction évoqués précédemment relèvent de cette volonté purement utilitariste.

Les études et enquêtes permettent aux médias de mieux connaître les particularités de leur audience existante ou attendue et d'adapter le produit en conséquence.

La publication dans le média de messages en provenance des usagers peut surtout s'avérer un moyen de nourrir la masse de contenus du média, et non se présenter comme l'ébauche d'un mécanisme de dialogue entre le pôle des usagers et le pôle production. Dans certains cas, ces publications relèvent même d'une obligation (morale ou légale) à laquelle le média se soustrairait volontiers.

Au mieux, certains procédés de feedback sont institutionnalisés afin de valoriser le destinataire du média dans sa qualité d'usager du produit. Ils lui confèrent ainsi un statut qui le rendra plus intimement lié au média et le placera vis-à-vis de lui dans une situation de plus grande dépendance.

Dans une certaine mesure, le même but "utilitariste" peut être attribué à l'ensemble des mécanismes mis en place par le média pour élaborer et gérer sa responsabilité sociale via certaines rubriques ou émissions ou par le truchement du travail d'un médiateur ou d'un ombudsman.

\section{Rétroactions négatives et perversions du feedback}

De manière globale, en s'inspirant de Bateson, on se permettra de qualifier l'ensemble des mécanismes évoqués ici de "feedback négatif".

1 Cl.-J. Bertrand, dossier "Déontologie des médias", Médiaspouvoirs, Paris, 1998 $n^{\circ} 4$, p. 54 . 
Et on constatera qu'une part importante des rétroactions relevées dans les systèmes médiatiques est de nature négative : suite à une erreur ou à un dysfonctionnement du média, ses usagers réagissent sous forme de courrier, télécopies, emails ou coups de téléphone. Ils lui demandent de modifier son opinion, de corriger une erreur ou d'apporter des précisions. La prise en compte de ces éléments permet au système médiatique de retrouver l'équilibre. En fonction de la théorie évoquée précédemment, il s'agit donc bien de feedback négatif. On considérera de même toutes les procédures apparentées au dépôt de plainte, qu'il s'agisse de mécanismes internes traditionnels, d'une partie du travail de l'ombudsman ou d'une part importante de la fonction d'instances externes ou semi-externes comme les Conseils de Presse ou les Conseils de régulation médiatique. Enfin, cette catégorie de "feedback négatif" comprendra aussi au moins une partie des systèmes d'étude et de mesure de "l'état" des usagers que constituent les enquêtes, sondages et études marketing. En effet, en menant ces recherches, l'entreprise entend s'adapter aux attentes (ou à l'évolution des attentes) de ses usagers et se rapprocher ainsi de son niveau d'équilibre.

On considérera de même comme feedback négatifs ceux que l'entreprise médiatique se voit obligée de prendre en considération, ou ceux qu'elle met en œuvre dans le seul but de légitimer sa propre existence.

Certains auteurs et professionnels de l'information estiment en effet que la mise en place d'interfaces de réception de rétroactions publiques ne peut se concevoir qu'en termes d'alibi pour un système médiatique "qui entretient l'illusion d'une interactivité toujours possible auprès de son public" ", La circularité serait en quelque sorte illusoire. Et, de nos jours, l'écran de fumée qui l'accompagnerait serait particulièrement convaincant, suite à l'impression de revalorisation des phénomènes de rétroaction qu'inspire le développement récent d'une multiplicité des supports interactifs favorisés par les progrès technologiques.

Même s'ils se parent de nombreux nouveaux atours, les systèmes de rétroaction ne continueraient-ils en définitive qu'à servir les intérêts de l'entreprise médiatique et ses souhaits d'adaptation à son environnement? La logique du binôme "fournisseur/consommateur"

\footnotetext{
'MATHIEN M., Le système médiatique, le journal dans son environnement, Paris, Hachette, 1989, p. 191.
} 
serait-elle implacable ? Déjà, naturellement, la différence de statut entre récepteur d'information et client est "souvent entretenue par les destinataires eux-mêmes qui, en réagissant à la diffusion d'une nouvelle, par courrier, par exemple, n'omettent pas de rappeler leur fidélité au journal, à la station de radio ou de télévision, et qu'ils ont la possibilité d'y mettre fin s'ils ne l'ont pas déjà fait au moment où ils ont rédigé leur lettre"'.

Dans ce cadre, serait-il finalement vain d'évoquer "le retour de l'usager" dans le processus médiatique?

\section{Pour des rétroactions positives}

Nous pensons que non. Même si la structure du système médiatique n'incite pas les individus-récepteurs traditionnels à réclamer une reconnaissance dans la sphère publique, même si l'interaction ne semble pas constituer actuellement un des buts recherchés par l'entreprise médiatique, il nous semble que la question de la rétroaction entre usagers et médias ne peut être abordée d'un seul point de vue utilitariste, s'inspirant -même sous le couvert de références systémiques- d'une lecture linéaire du processus de communication. Notre vision plaide pour une reconnaissance à part entière de l'usager par l'entreprise médiatique, non en tant qu'objet de communication mais comme un véritable sujet communiquant, pouvant prendre part à un dialogue avec le média, voire, dans les meilleurs des cas, en devenir un partenaire.

Nous venons de l'évoquer longuement : la logique de fonctionnement du système médiatique (comme de tous les systèmes) étant de tendre à l'homéostasie, les systèmes de feedback concourant à cette prise en compte "positive" de l'utilisateur du média s'avèrent rares. Néanmoins, ils existent et constituent, dans la foulée de Bateson, de véritables modes de "feedback positif".

Dans un premier temps, on considérera ici comme tel tout mode de feedback poussant à une interpellation, une mise en cause du média, c'est-à-dire à sa déstabilisation. Pour autant que le média en tienne compte, on inscrira essentiellement dans cette catégorie une partie du courrier qui lui est adressé, de même que les contenus véhi-

1 M. MATHIEN op. cit., 1989, p. 193. 
culés par les autres modes de feedback classiques. L'ensemble des initiatives extérieures poussant le média au changement figureront aussi, naturellement, parmi les modes "positif" de feedback.

Mais, dans le contexte d'une revalorisation du pôle "usagers" pour laquelle nous nous prononçons depuis le début de cet exposé, nous souhaiterions également conférer à cette notion de "feedback positif" un spectre plus large que celui qui lui est attribué par la catégorisation de Bateson. Au-delà de tout processus mettant l'entreprise médiatique en situation de déséquilibre, nous proposerons de considérer ici comme "positif" tout mode de feedback qui ne se positionne pas en rétroaction vis-à-vis du système-média, mais bien en action (positive). Que l'initiative en soit prise par le média lui-même ou par ses usagers. Le "feedback" positif sera ainsi considéré comme celui qui contribue à une reconnaissance active de l'usager en tant que sujet prenant part de la manière la plus complète possible au déroulement intégral du processus de communication. Ainsi en sera-t-il, par exemple, de toute initiative permettant aux usagers de s'exprimer et de participer à l'élaboration du média, à différents niveaux. Si, dans certains cas, la simple et traditionnelle rubrique du courrier des lecteurs peut déjà être classée dans cette catégorie, celle-ci comprendra aussi, par exemple, les cas de "journal des usagers", espace du journal mis à disposition des lecteurs, dont parle Michel Mathien! Tout comme, autre exemple, l'expérience de participation des lecteurs à la rédaction de l'édition locale de Guebwiller du journal L'Alsace à en 1979, également relatée par Mathien². Un système interactif qui avait aussi, notamment, été tenté par France 2 (portant alors le nom de Antenne 2) après mai 1981 au cours de ses journaux télévisés de week-end.

Il en sera de même de tout établissement d'un réel dialogue (ou d'une ébauche de dialogue) entre média et usagers, que cet échange ait lieu au sein du média lui-même, de ses extensions (par exemple, via les forums des sites Internet) ou lors de rencontres interpersonnelles.

\footnotetext{
1 M. Mathien Les journalistes et le système médiatique, Paris, Hachette, 1992, p. 231.
}

2 Ibid., p. 232. 


\section{Un nouveau pacte communicationnel}

Même si les agents de résistance au changement semblent en l'état encore dominer la situation, de nombreux indices plaident aujourd'hui pour que soient redéfinies les clauses du "contrat communicationnel" qui lie, quoi qu'il arrive, émetteur et usager de messages véhiculés par des entreprises médiatiques. Au-delà d'une cynique utilisation des feedback dans le seul but d'auto-entretenir le fonctionnement d'un système-médias, la lente émergence d'une culture sociale mettant en exergue les valeurs de citoyenneté impose que ces rapports finissent par être abordés sous un jour neuf. Déjà, les outils de communication mis à la disposition des usagers par les nouvelles technologies de l'information modifient, au quotidien, les systèmes de relations entre lecteurs, auditeurs, téléspectateurs, et instances de production des messages. Davantage critiques vis-à-vis des médias, des composantes du "public" souhaitent désormais en être mieux reconnues. Ces revendications, ou les évolutions naturelles de la société, aboutiront-elles à l'émergence de médias où règnera un état de partenariat entre producteurs et récepteurs de la chose médiatique ? Dans un univers reposant sur l'industrialisation de la communication, cette étape paraît, au moins à l'heure actuelle, utopique. Mais, comme nous en avons tenté l'ébauche dans les lignes qui précèdent, il semble acquis que l'ancestrale distance entre les médias et leurs utilisateurs appartient désormais à un passé révolu. Petit à petit se dessinent ainsi les contours d'un nouveau "pacte communicationnel" qui permettrait de redéfinir la nature des relations entre les différents acteurs du processus médiatique, en prenant tant en compte le besoin de reconnaissance des usagers que celui des producteurs de messages, qui veillent depuis toujours à la qualité du processus.

Il ne s'agit pas ici de renverser le sens des mécanismes de la communication, comme le prônait notamment jadis Enzenberger ${ }^{1}$ à la suite de Berthold Brecht ${ }^{2}$. Il ne s'agit pas davantage de communier sans partager aux constats qu'établissait Baudrillard à l'époque de mai '68 lorsqu'il déplorait que "ce qui caractérise les médias de masse,

1 H.M. EnZEnBERGER, "Constituents of a theory of the media", in D. MC QuAIL (ed.), Sociology of Mass Communications, Harmondsworth, Penguin, 1972.

2 B. BRECht, Théorie de la radio, Paris, L'Arche, 1970. 
c'est qu'ils sont anti-médiateurs, intransitifs, qu'ils fabriquent de la non-communication -si on accepte de définir la communication comme un échange, comme l'espace réciproque d'une parole et d'une réponse, donc d'une responsabilité,- et non pas une responsabilité psychologique et morale, mais une corrélation personnelle de l'un et de l'autre dans l'échange"l. Baudrillard écrivait à l'époque que les médias de masse s'étaient "interdit à jamais la réponse", ce qui rendait impossible tout procès d'échange.

Les choses ont aujourd'hui quelque peu changé. Les partenaires existent et se parlent. Il reste à formaliser leurs relations.

1 J. Baudrillard, Pour une critique de l'économie politique du signe, Paris, Gallimard, 1972, p. 208. 